Peter coss, The Aristocracy in England and Tuscany, 1000-1250

\title{
Enrico Faini
}

\section{(2) OpenEdition}

\section{Journals}

Édition électronique

URL : https://journals.openedition.org/ccm/8751

DOI : $10.4000 / \mathrm{ccm} .8751$

ISSN : 2119-1026

\section{Éditeur}

Centre d'études supérieures de civilisation médiévale/Université de Poitiers

\section{Édition imprimée}

Date de publication : 1 décembre 2021

Pagination : $385-388$

ISBN : 978-2-490783-11-3

ISSN : 0007-9731

\section{Référence électronique}

Enrico Faini, «Peter coss, The Aristocracy in England and Tuscany, 1000-1250 ", Cahiers de civilisation médiévale [En ligne], 256 | 2021, mis en ligne le 01 décembre 2021, consulté le 01 juin 2022. URL: http://journals.openedition.org/ccm/8751; DOI : https://doi.org/10.4000/ccm.8751

\section{(2) $\odot \Theta \Theta$}

La revue Cahiers de civilisation médiévale est mise à disposition selon les termes de la Licence Creative Commons Attribution - Pas d'Utilisation Commerciale - Pas de Modification 4.0 International. 
Peter Coss, The Aristocracy in England and Tuscany, 1000-1250, Oxford, Oxford University Press, 2020.

Comme l'auteur le soutient à plusieurs reprises, ce livre n'est pas un essai d'histoire comparée (p. 1,271 et 442). Mais alors à quoi bon écrire près de 500 pages sur l'aristocratie médiévale, dont la moitié est consacrée à l'étude de la Toscane et un tiers à celle de l'Angleterre? Pour comprendre la raison de ce formidable ouvrage, nous devons partir des deux points d'arrivée différents qui caractérisent l'histoire toscane et anglaise entre le $\mathrm{XI}^{\mathrm{e}}$ et le XIII ${ }^{\mathrm{e}} \mathrm{s}$. Dans les deux cas, ces acquis du $\mathrm{XIII}^{\mathrm{e}} \mathrm{s}$. ont conditionné la manière de lire les phénomènes précédents : la commune dans le cas de la Toscane, la Magna Carta et le parlementarisme dans le cas de l'Angleterre. Il est donc nécessaire de relire les deux mythes historiographiques à la lumière d'une meilleure connaissance des contextes. Cette perspective - qui ne peut être définie "comparative", mais simplement "plus large" ou "plus profonde" - permet d'identifier de nombreux points de contact entre les deux histoires et pourrait inaugurer une saison historiographique moins conditionnée par les traditions nationales.

L'aristocratie anglaise est un thème cher à l'auteur, qui a consacré une monographie à la noblesse dès 2003 (The Origins of the English Gentry, Cambridge, Cambridge University Press [Past and Present Publications], 2003). Étendre l'enquête à la période pré-normande s'inscrit donc dans une évolution naturelle des intérêts. Ceux qui étudient ces thèmes sont souvent confrontés au monde français et allemand, il est plus rare qu'ils portent leur regard sur le monde méditerranéen. Dans le contexte méditerranéen, la Toscane représente un cas d'étude idéal, c'est pourquoi l'auteur l'a choisie pour la comparaison : 
les enquêtes sur l'aristocratie sont nombreuses et de bon niveau et s'appuient sur une documentation exceptionnellement abondante et accessible. P. Coss s'adresse principalement au public anglais et c'est la raison pour laquelle l'histoire de la Toscane est résolument prépondérante dans le livre : non seulement le nombre de pages qui lui sont consacrées est plus grand, mais il a également une fonction heuristique précise, représentant en fait un modèle de comparaison fréquemment rappelé tout au long de la section consacrée à l'Angleterre.

Après un chapitre introductif, le contexte toscan est présenté. Au lendemain de la désintégration de l'empire carolingien, dans le Regnum Italicum (comprenant le centre et le nord de l'Italie) comme ailleurs, on assiste à la dissolution du publicum qui se fragmente en une série de dominatus locaux. En Toscane, cependant, les cadres institutionnels publics ont mieux résisté qu'ailleurs. En particulier, le marquis de Tuscia (Toscane) est resté le point de référence pour toute autorité locale jusqu'à la période de la querelle des Investitures (v. 1080 1120). Au cours de cette période, et pas seulement en Toscane, il y a eu une forte augmentation de l'instabilité politique et de la violence qui a conduit à une mutation du pouvoir. Comme le reste du Regnum Italicum, la Toscane (en particulier sa partie nord) était caractérisée par un réseau dense de villes. Dans la ville et le territoire environnant, au moins depuis le $\mathrm{X}^{\mathrm{e}} \mathrm{s}$., l'évêque était la figure de référence du point de vue politique; il n'est donc pas surprenant que l'aristocratie contrôle ses nominations et s'implique dans l'administration des biens épiscopaux. Mais à partir $\mathrm{du}$ milieu du $\mathrm{XI}^{\mathrm{e}} \mathrm{s}$., les mouvements de réforme de l'Église bouleversent l'équilibre politique dans lequel l'évêque représente une expression des pouvoirs locaux. Ces deux phénomènes (la Réforme de l'Église et la querelle des Investitures) ont profondément affecté les relations entre l'aristocratie et les villes.

$\mathrm{P}$. Coss s'attaque à ce problème en se concentrant sur le cas de Florence (mais les résultats peuvent également être étendus à d'autres villes) et en basant son exposition principalement sur les études de Maria Elena Cortese. M. E. Cortese a en effet identifié un niveau intermédiaire entre la grande aristocratie (celle qui a un titre d'origine publique comme comte ou marquis) et la très petite (titulaire de droits seigneuriaux sur un très petit territoire, tout au plus un château). C'est au niveau de l'aristocrazia intermedia (P. Coss maintient l'expression dans sa version originale en italien) que les changements les plus importants ont eu lieu. En fait, jusqu'aux premières décennies $\mathrm{du} \mathrm{XII}^{\mathrm{e}} \mathrm{s}$., cette aristocratie est restée ancrée à la résidence urbaine et ne s'est ruralisée qu'ensuite, à l'instar de la haute aristocratie qui l'avait fait un ou deux siècles plus tôt. Pour ceux qui étudient l'Italie de l'extérieur, c'est une acquisition importante, car elle brouille le modèle weberien selon lequel, dans le monde méditerranéen, la ville restait la résidence de l'aristocratie et représentait un obstacle à l'ascension d'autres groupes sociaux. Un autre élément qui caractérise l'aristocrazia intermedia est l'absence de normes privilégiant l'un des héritiers au détriment des autres. Cela a imposé le développement d'instruments juridiques pour la gestion partagée des biens et a fait du monde aristocratique toscan quelque chose de plus semblable aux communes (ou de moins dissemblable à celles-ci). Le thème est au cœur de la proposition interprétative de P. Coss : l'aristocratie a également expérimenté des formes institutionnalisées et sophistiquées d'alliance et de collaboration horizontale, ainsi que des relations de clientèle bien connues et étudiées.

Le détachement de l'aristocrazia intermedia de la ville a déterminé le développement, à partir du $\mathrm{XII}^{\mathrm{e}} \mathrm{s}$., d'une aristocratie urbaine aux caractéristiques nouvelles. L'auteur décrit son profil principalement à travers le cas de Pise. Parmi les villes italiennes, Pise jouit de nombreux records : une saison de construction luxuriante dès $1 \mathrm{e} \mathrm{XI}^{\mathrm{e}} \mathrm{s}$., une historiographie abondante et précoce, une documentation des institutions municipales déjà avant 1100 . À l'origine de ces performances, il y a le commerce international qui a certainement enrichi le groupe dirigeant de la cité, le rendant moins lié à la terre que dans le reste de la Toscane (et la plupart de l'Occident). Cela a fait de la ville un environnement attractif également pour la haute aristocratie. Mais la dimension territoriale de la domination aristocratique s'exprime aussi très bien dans le tissu urbain, où les églises familiales, les quartiers fortifiés et les tours ont décliné de façon originale l'aspiration au dominatus local.

Qu'est-il arrivé à la haute aristocratie dans les autres villes toscanes? Les grandes lignées de comtes de la Toscane orientale et méridionale n'avaient aucun intérêt à revenir dans les villes qui ne pouvaient offrir aucune perspective particulière d'enrichissement. Jusqu'à une époque assez récente, le monde des comtes toscans était considéré comme un reliquat négligeable : une série de châteaux attendant d'être balayés par la glorieuse avancée de la puissance urbaine. Aujourd'hui, la position de l'historiographie est plus nuancée. Guidi et Aldobrandeschi, par exemple, ont intercepté les ressources de l'espace agraire en expansion à la fois par un prélèvement seigneurial efficace et par des initiatives de 
construction dédiées à la concentration des hommes. Plutôt que des batailles d'arrière-garde, des initiatives assez ambitieuses qui ont permis à ces groupes aristocratiques une très large liberté de manœuvre politico-militaire, dans laquelle la violence exercée sur les sujets et sur les autres seigneurs représentait un ingrédient essentiel, également pour définir le status. L'exercice de la violence au cours du XII ${ }^{\mathrm{e}} \mathrm{s}$. constituait un canal de promotion sociale efficace, si efficace qu'il fallait le discipliner pour ne pas compromettre le mode de vie des groupes dominants. C'est dans ce contexte que la chevalerie a été établie comme une idéologie basée sur un rite sélectif (l'adoubement). Le barrage a induit l'action politique organisée de tous ceux qui étaient exclus : le Popolo (en italien). Ce mouvement, vu aujourd'hui principalement à travers le prisme de l'historiographie sur les villes d'Italie ou des Pays-Bas, a au contraire - dit l'auteur une utilité plus générale et permet une lecture plus complexe des événements qui ont touché l'Angleterre dans les premières décennies du XIII $\mathrm{s}$.

L'historiographie anglaise a longtemps concentré son intérêt sur les structures de pouvoir liées à la monarchie. James Campbell n'a pas hésité à parler des États-nations en regardant le royaume anglo-saxon au début du deuxième millénaire. Il est clair que ce n'est pas un État au sens moderne du terme, mais plutôt un État comme la monarchie carolingienne aurait pu l'être. La haute aristocratie restait compacte autour du souverain car, au moins en partie, elle dépendait de sa faveur : ce concept (Königsnähe en allemand) est précisément importé de l'étude du monde carolingien. Cela ne signifie pas que cette même aristocratie (les earls ou, à un niveau inférieur, les thegns) ne possédait pas déjà une indépendance considérable au niveau local ; par exemple : la construction de résidences fortifiées, les burhs, ressemble au processus d'incastellamento que l'on rencontre en Toscane (même si elle ne possède pas cette intensité). Même dans la relation entre l'aristocratie anglo-saxonne et les centres urbains, nous trouvons des similitudes avec la Toscane : dans les deux cas, il y avait une forte interconnexion, ce qui a entraîné des efforts pour dominer de grands secteurs du tissu urbain. Au milieu du XI ${ }^{\mathrm{e}}$ s., en Toscane et en Angleterre, le lien avec le roi reste déterminant plus sur le plan de la légitimation du pouvoir que sur celui de son exercice effectif.

Avec cette prise de conscience, il est possible de lire en termes moins téléologiques et dichotomiques les événements du royaume, d'abord sous la dynastie anglo-normande, puis sous les Plantagenêts : la téléologie de la conclusion "monarchique" et la dichotomie entre public et privé. S'il est vrai que la monarchie et l'aristocratie ont pu trouver un équilibre, il est tout aussi vrai que l'exercice de la justice a été géré de plus en plus de manière autonome par les nobles, à la fois par le biais des tribunaux seigneuriaux, et par ceux détenus au nom du roi. L'étude de cas des comtes de Chester montre comment le succès (ou l'échec) d'une affirmation aristocratique, encore au milieu du $\mathrm{XIII}^{\mathrm{e}} \mathrm{S}$., est resté lié à la capacité d'être au four et au moulin : le four de la faveur royale et le moulin du pouvoir local. Ce pouvoir $-P$. Coss est très clair sur ce point - peut être comparé à celui des comtes Guidi ou Aldobrandeschi en Toscane (p. 399) : il s'agit avant tout d'une suggestion à l'historiographie anglaise, qui tend à surestimer le contrôle territorial de la monarchie. Cependant, il est évident que l'auteur pense à un autre scénario possible pour l'histoire de la Toscane, très différent de celui plus habituel des communes et des villes.

Dans les ouvrages consacrés au XIII ${ }^{\mathrm{e}} \mathrm{S}$, l'aristocratie joue deux rôles apparemment opposés : en Toscane celui de l'antagoniste perdant face au triomphe de la commune, en Angleterre celui du protagoniste gagnant contre la tyrannie monarchique. Comme le montre de façon convaincante l'auteur, ces deux rôles sont en grande partie le résultat d'une illusion d'optique et de distinctions erronées : si nous étendons notre regard aux trois siècles précédents, le monde aristocratique s'avère protéiforme, résilient de la même façon face aux nouvelles sollicitations. Le même phénomène des communes, en fait, peut être $l u$ - sinon à travers le prisme de la patrimonialisation des pouvoirs du moins à travers celui de leur territorialisation; après tout est une erreur : opposer trop rigidement les relations horizontales des communes aux relations verticales de l'aristocratie, comme l'a fait remarquer Susan Reynolds il y a plus de vingt ans. D'autre part, derrière la Carta plus ou moins extorquée à Jean sans Terre, il y avait des barons assiégés par une série de petites instances (l'équivalent anglais de l'aristocrazia intermedia toscane, mais aussi les communautés), ce qui le fait ressembler davantage à un compromis, temporaire et prudent, qu'à un traité “constitutionnel". Que le pouvoir central soit absent (dans le cas des communes italiennes) ou qu'il soit soudainement devenu trop envahissant (dans le cas de l'aristocratie face à Jean sans Terre), à la base du protagonisme des pouvoirs moyens (communes ou aristocratie) il y avait un vide du pouvoir central (p. 416).

Il est difficile de trouver des limites dans un ouvrage qui montre la même facilité à naviguer entre des bibliographies aussi vastes que divergentes : on peut plutôt trouver des points sur lesquels il serait bon de 
discuter dans d'autres recherches futures. S'il est vrai qu'au niveau de la domination territoriale, les phénomènes de commune et de seigneurie sont similaires, nous ne pouvons pas oublier que dans les communes, la gestion collective du pouvoir a atteint un niveau de sophistication qu'il était difficile de trouver dans la documentation contemporaine des dominations seigneuriales : la conservation documentaire n'est pas seulement une question de hasard ou de scrupule administratif, mais aussi une demande précise de transparence, utile au niveau du consensus; il pourrait donc être fructueux d'axer les futures recherches sur cette question. En outre, aux yeux d'un universitaire formé sur la rive méditerranéenne, peut sembler un peu rigide l'utilisation de distinctions qui, dans le contexte italien, sont considérées comme une nuance sociale : haute aristocratie, capitanei/aristocrazia intermedia, secundi milites/valvassores/lambardi. Il s'agit cependant de vérifier si derrière ces termes il y a vraiment des éléments communs, liés à des pratiques coutumières répandues en Occident et sur la dimension unificatrice desquels nous commençons à réfléchir. En revanche, dans le contexte anglais, quelqu'un pourrait être surpris parce que l'auteur a adopté un point de vue "méditerranéen", dans lequel certaines distinctions sont trop lâches. Le livre suscitera probablement un débat sur l'utilité d'adopter, après les recherches de Pierre Toubert, d'autres catégories interprétatives à partir des exemples de l'Italie centrale (l'aristocrazia intermedia).

Les 36 pages de bibliographie - une longue série de titres tous largement présentés et vivement discutés dans le volume - témoignent de la qualité de la recherche. Un livre généreux et courageux, celui de P. Coss, qui influencera certainement les futures études sur l'aristocratie européenne. Même si, fidèle à son understatement, l'auteur évite de le dire en termes trop tranchés, le livre représente un coup bien placé contre les reconstructions qui se plaisent à des spécificités locales et des horizons limités.

Enrico FAINI

Université de Florence 